\title{
S13-6
}

\section{Correction of Penile Curvature}

Professor Urology and Pediatrics, Chief Pediatric Urology, UCSF

Laurence S. Baskin

\section{Etiology of Penile Curvature}

Skin

Dartos Fascia

Corporeal Disproportion

Short Urethral

\section{Prevalence of Curvature}

With Hypospadias

More prevalent with severe cases

Estimate $15-20 \%$ require procedures other than skin/dartos takedown

Without Hypospadias

\section{Concepts of the Urethral Plate}

Preservation When Possible

Allows onlay flap reduces stenosis

Incision and tubularization possible

Rarely causes curvature resection requires tube versus 2 stage

\section{Techniques for Repair \\ Concepts \\ Preserve Nerves \\ Anatomically based \\ Simple \\ Durability}

Extensive skin and dartos dissection

Tunica Albuginea Plication (TAP)

\section{Midline Dorsal Plication}

Preferred technique

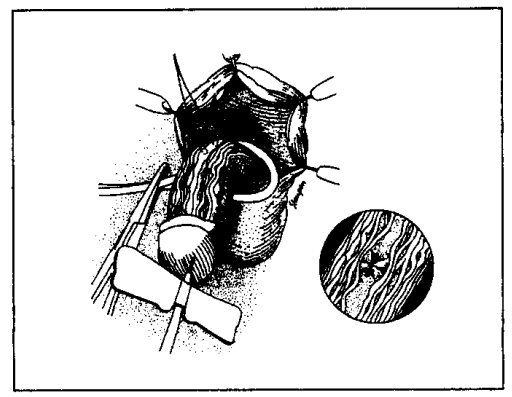

\section{Dermal Grafts}

Severe curvature

Resection of the urethral plate

Two stage repair 